\title{
Quantum Monte Carlo estimators for the positron-electron annihilation rate in bound and low-energy scattering states
}

\author{
Simone Chiesa* \\ Department of Physics, University of Illinois Urbana-Champaign, Urbana-Champaign, Illinois 61801, USA \\ Massimo Mella ${ }^{\dagger}$ \\ Central Chemistry Laboratory, Department of Chemistry, University of Oxford, South Parks Road, Oxford OX1 3QH, United Kingdom \\ Gabriele Morosi \\ Dipartimento di Scienze Chimiche, Fisiche e Matematiche, Università dell'Insubria, via Lucini 3, 22100 Como, Italy
}

(Received 12 September 2003; published 2 February 2004)

\begin{abstract}
Variational and exact estimators for the positron-electron annihilation rate in bound states of systems containing a positron in the framework of quantum Monte Carlo methods are presented. The modification needed to compute the effective number of electrons $Z_{\text {eff }}$ when scattering states are concerned is also discussed. The algorithms are tested against four cases for which close to exact results are available, finding an overall good agreement. The systems are $\mathrm{Ps}^{-}, \mathrm{PsH}$, and the $s$-wave scattering component of $e^{+} \mathrm{H}$ and $e^{+} \mathrm{He}$.
\end{abstract}

DOI: 10.1103/PhysRevA.69.022701

PACS number(s): 34.85.+x, 02.70.Ss, 36.10.Dr

\section{INTRODUCTION}

One of the most intriguing features of the physics of positronic systems is, with no doubts, the possibility of an annihilation event. The two or more photons emerging from this process carry information about the electronic structure of the system under study and can be analyzed by means of different experimental techniques (see Ref. [1] for a general discussion of these techniques).

In gases, some of the salient features of this phenomenon have been recently clarified from the experimental as well as the theoretical side. For example, the possibility of producing highly monochromatic positron beams has allowed to directly observe the role played by Feshbach resonances in the annihilation process in molecular gases [2]. Even more recently the behavior of the annihilation cross section close to the positronium formation threshold has been clarified correcting it for the finite lifetime of Ps [3]. The most important consequence of this correction is that the annihilation cross section does not diverge at the Ps formation threshold [3,4], but instead interpolates smoothly between the values it would get from a purely nonrelativistic treatment. This behavior has been also confirmed by Igarashi et al. [5] who investigated the effect by introducing an absorbing potential into the scattering Hamiltonian to take positron annihilation into consideration [6].

The annihilation properties of bound states of electronic systems containing a positron can also be relevant to understand the experimental results in those cases where the lifetime of the antiparticle is longer than the time required for it to thermalize. In this respect quantum Monte Carlo (QMC) methods have already proved themselves to be an easy and powerful way to get accurate properties for many-electron

\footnotetext{
*Electronic address: chiesa@uiuc.edu

†Electronic address: Massimo.Mella@chem.ox.ac.uk

‡Electronic address: Gabriele.Morosi@uninsubria.it
}

systems containing a positron [7-12].

The main goal of this paper is to present an exact estimator for the annihilation rate in bound states and in low-energy scattering processes in the stochastic framework of Monte Carlo methods. The paper is organized as follows. In Sec. II the basic definition of the annihilation rate and the notation used throughout the paper are introduced. In Sec. III the form of the estimators used in simulations are reported and in Sec. IV they are applied to bound and scattering states. Specifically we considered the ground state of $\mathrm{PsH}$ and $\mathrm{Ps}^{-}$ and the $s$-wave component for the scattering of a positron off hydrogen and helium. In Sec. V the algorithm is briefly compared with other approaches.

\section{DEFINITIONS AND NOTATION}

Let us first introduce a functional $w$ defined as

$$
\begin{aligned}
w[\Psi]= & \sum_{i=1}^{N} \int d \mathbf{r}_{1} d \mathbf{r}_{2} \cdots d \mathbf{r}_{N} \\
& \times\left|\Psi\left(\mathbf{r}_{1}, \mathbf{r}_{2}, \ldots, \mathbf{r}_{i}, \ldots, \mathbf{r}_{p}=\mathbf{r}_{i}\right)\right|^{2},
\end{aligned}
$$

where $\Psi$ is a many-body eigenfunction of the nonrelativistic Hamiltonian describing a Coulomb system composed of $N$ electrons $\left(\mathbf{r}_{1}, \mathbf{r}_{2}, \ldots, \mathbf{r}_{N}\right)$ and one positron $\left(\mathbf{r}_{p}\right)$. When bound states are concerned, $w$ is related to the two-photon annihilation rate $\Gamma_{2 \gamma}$ by [13]

$$
\Gamma_{2 \gamma}=\pi \alpha^{4} c a_{0} w[\Psi]
$$

where $\alpha$ is the fine-structure constant, $c$ the light speed, and $a_{0}$ the Bohr radius. $\Psi$ is normalized according to $\int d \mu|\Psi(x)|^{2}=1, d \mu$ being the measure in the entire configurational space and $x$ the set of all particle coordinates. Let us define as $x_{\mathrm{el}}$ the set of all electron coordinates, $x$ $=\left(x_{\mathrm{el}}, \mathbf{r}_{p}\right)$, and $d \nu$ the measure in the electronic subspace, 
the two measures being related by $d \mu=d \nu d \mathbf{r}_{p}$. To shorten the formulas we define a new function

$$
\Phi^{(i)}\left(x_{\mathrm{el}}\right)=\Psi\left(x_{\mathrm{el}} ; \mathbf{r}_{p}=\mathbf{r}_{i}\right) .
$$

With this notation $w$ can be rewritten as

$$
w[\Psi]=\sum_{i=1}^{N} \int d \nu\left|\Phi^{(i)}\right|^{2} .
$$

In Sec. IV results will be presented in terms of the average value of the Dirac $\delta$ operator $\left\langle\delta_{e p}\right\rangle$ which can be defined as $\left\langle\delta_{e p}\right\rangle=w / N$ for the simple systems treated here.

Possible ways to calculate this quantity in the computational framework of QMC methods have been already proposed [14]. They range from a direct computation of the integral in Eq. (3) [15] to techniques based on extrapolation [14] of a nonlocal estimator of the Dirac $\delta$ function [16]. The latter have the great advantage of being computationally very light and straightforward to implement, but are plagued by a large variance of their estimator. To avoid this problem, the present method is close in spirit to the first of these approaches.

As for scattering experiments, the quantity of interest is the effective number of electrons $Z_{\text {eff }}$, which is defined as $w$ in Eq. (1), $\Psi$ being a scattering eigenstate describing an asymptotic density of one positron per unit volume. This condition is sometimes expressed by the relation

$$
\Psi(x) \rightarrow \Psi_{\mathrm{el}}\left(x_{\mathrm{el}}\right) e^{i \mathbf{k} \cdot \mathbf{r}_{p}}, \quad r_{p} \rightarrow \infty
$$

where $\Psi_{\mathrm{el}}$ is the normalized electronic wave function and $\mathbf{k}$ is the positron momentum. The annihilation cross section $\sigma$ is related to the effective number of electrons $Z_{\text {eff }}$ through the relation [17]

$$
\sigma=\frac{\pi c r_{0}^{2}}{v} Z_{\mathrm{eff}},
$$

where $v$ is the positron velocity and $r_{0}$ the classical electron radius.

\section{QMC ESTIMATE}

This section contains the technical description of the QMC estimate of $w$. The algorithm necessary to extract this quantity for bound states by variational Monte Carlo (VMC) and diffusion Monte Carlo (DMC) within the fixed-node approximation (Secs. III A and III B) are presented. The necessary modifications for scattering states are discussed in Sec. III C. In the following we will refer to the fixed-node approximation as exact, meaning that the Schrödinger equation is solved exactly within the nodal boundary defined by the trial wave function $\Psi_{T}$. Indeed, for the one- and twoelectron systems treated in this paper the wave functions are nodeless and therefore no fixed-node approximation has to be invoked. For the sake of brevity only one of the addends of Eq. (3) will be considered and $w$ will be defined consequently. The extension to the whole sum is straightforward.

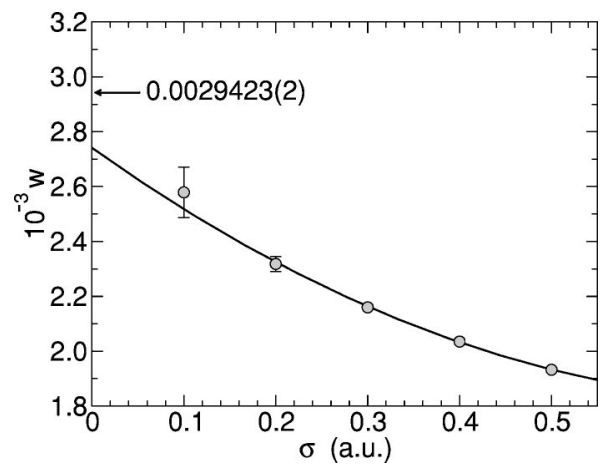

FIG. 1. Comparison of the variational estimator [Eq. (7), arrow] with a technique based on the extrapolation from a sequence of Gaussians of decreasing width $\sigma$ [14] (circles; the continuous line is a second-order polynomial fitted to these values). The system considered is $e^{+} \mathrm{H}$ with $\mathcal{R}=10 \mathrm{bohr}$ (see text for the definition of $\mathcal{R}$ ). The two estimates were computed during the same simulation. The error bar of the new estimator is smaller than the line used to represent the arrow.

\section{A. Variational estimate}

To illustrate how to get an efficient estimator for $w$ let us begin with introducing a function $\xi$ such that

$$
1=\mathcal{N}^{2} \int \xi\left(\mathbf{r}_{p}\right)^{2} d \mathbf{r}_{p} .
$$

Multiplying Eq. (3) by Eq. (6) and rearranging the numerator of the right-hand side of the obtained expression allows us to rewrite the variational expectation value of the annihilation rate as

$$
w\left[\Psi_{T}\right]=\mathcal{N}^{2} \frac{\int d \mu\left|\frac{\Phi_{T} \xi}{\Psi_{T}}\right|^{2}\left|\Psi_{T}\right|^{2}}{\int d \mu\left|\Psi_{T}\right|^{2}} .
$$

This quantity can be easily computed during a VMC simulation. It is important to note that we could have chosen as well a normalizable function $\xi^{\prime}\left(\mathbf{r}_{1 p}\right)$ (i.e., a function of the relative positron-electron positions instead of a function of the positron position) without spoiling the exactness of the approach. As commented later, both functions $\xi\left(\mathbf{r}_{p}\right)$ and $\xi^{\prime}\left(\mathbf{r}_{1 p}\right)$ were found adequate for scattering states, but $\xi^{\prime}\left(\mathbf{r}_{1 p}\right)$ leads to much smaller error bars in ground-state calculations. The precise form of $\xi$ and $\xi^{\prime}$ is specified in Sec. IV. It is worth stressing that $\xi$ and $\xi^{\prime}$ are functions of three coordinates, so an accurate computation of the normalization constant $\mathcal{N}$ is always feasible. This allows for a certain freedom in the choice of their analytical form and this freedom can be exploited to lower the variance of this estimator.

As an introductory example of the usage of Eq. (7), for the $e^{+} \mathrm{H}$ system in Fig. 1 this estimator is compared with the technique based on the extrapolation of the average value of a set of Gaussians over the sampled distribution [14].

Let us finally remark that an accurate variational estimate of any property normally involves the extensive optimization of the parameters defining the wave function. However, the 
extremely local character of the computed quantity poses serious problems on the quality of the final results. Optimization is usually carried out minimizing the variance or the energy of the trial wave function. These properties as well as any linear combination of them are global properties which depend on the entire distribution and not on the details of the wave function in small regions of the configurational space [18]. These are scarcely weighted and somehow neglected by the optimization process.

\section{B. Exact estimate}

It is for the reasons outlined at the end of the preceding section that an exact Monte Carlo estimate can be useful. Because the analytical form of the exact eigenfunction $\Psi_{0}$ is not known, there is no way to exploit directly the simple estimator provided in Eq. (7). However, $w\left[\Psi_{0}\right]$ can be decomposed as

$$
w\left[\Psi_{0}\right]=\frac{\int d \nu\left|\Phi_{0}\right|^{2}}{\int d \nu\left|\Phi_{T}\right|^{2}} \frac{\int d \nu\left|\Phi_{T}\right|^{2}}{\int d \mu\left|\Psi_{T}\right|^{2}} \frac{\int d \mu\left|\Psi_{T}\right|^{2}}{\int d \mu\left|\Psi_{0}\right|^{2}},
$$

where one recognizes the central ratio as the one provided by the variational calculation. So, the exact estimate of $w$ can be obtained as long as the remaining ratios are exactly computed. The quantity $u(x)=\Psi_{0}(x) / \Psi_{T}(x)$, routinely computed in the forward walking algorithm, provides a straightforward solution to this problem. Using it, the product of the two ratios can be rewritten as $\mathcal{U} \mathcal{V}^{-1}$, where

$$
\mathcal{U}=\frac{\int d \nu u^{2}\left|\Phi_{T}\right|^{2}}{\int d \nu\left|\Phi_{T}\right|^{2}}, \quad \mathcal{V}=\frac{\int d \mu u^{2}\left|\Psi_{T}\right|^{2}}{\int d \mu\left|\Psi_{T}\right|^{2}} .
$$

For the sake of completeness a brief explanation of the steps leading to the estimator for $u$ is presented. First let us define $P_{\beta}(y)$ as

$$
P_{\beta}(y)=\int d x G^{T}(y, x, \beta)
$$

where $G^{T}$, the importance sampling Green's function, is defined starting from the imaginary time Green's function $G(y, x, \beta)$ as

$$
G^{T}(y, x, \beta)=\frac{\Psi_{T}(x)}{\Psi_{T}(y)} G(y, x, \beta) .
$$

Expanding $G$ in its normalized eigenstates $\phi_{n}$ and taking $\beta$ $\gg 1$ leads to

$$
P_{\beta}(y)=e^{-\beta\left(E_{0}-E_{r}\right)} \frac{\phi_{0}(y)}{\Psi_{T}(y)} \int d x \phi_{0}(x) \Psi_{T}(x),
$$

where $\phi_{0}$ is the ground-state wave function. Hence $u(y)$ is simply proportional to $P_{\beta}(y)$ for large values of $\beta$. Finally, a way to compute $P_{\beta}$ becomes manifest inserting the func-
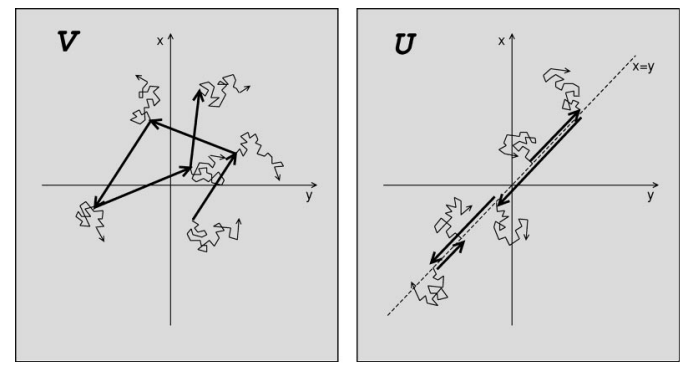

FIG. 2. Schematic representation of the algorithms to compute $\mathcal{V}$ and $\mathcal{U}$. A two bodies one-dimensional system is considered. $x$ and $y$ can be regarded as the coordinates of the electron and the positron. Simulation is carried out with a single walker. Thick lines are variational paths, while the thin ones are diffusion paths. The left graph schematizes the case when one samples $\Psi_{T}$, the right graph the case when one samples $\Phi_{T}$, that is, positron and electron are always on top of each other in the variational random walk.

tional integral form of the importance sampling Green's function [19] in Eq. (10). This leads to

$$
\int_{y \rightsquigarrow} \mathcal{D}^{T} X \mathcal{W}(X)=P(y),
$$

where $X$ has been used to indicate a path. $\mathcal{D}^{T} X$ represents the functional measure associated with $\Psi_{T}$ and $\mathcal{W}$ is defined by $\mathcal{W}=\exp \left(-\int E_{l o c} d x\right)$. The notation " $y \rightsquigarrow$ " stands for "all paths generating from $y$." In principle $\Psi_{0} / \Psi_{T}$ should be computed as the average weight $\mathcal{W}$ over the set of infinitely long paths generating from $y$. In practice the weight over a single long enough chain provides an unbiased estimate.

The algorithm for the computation of the two ratios consists therefore in (a) sampling $\left|\Psi_{T}\right|^{2}$ or $\left|\Phi_{T}\right|^{2}$ and (b) computing the ratio $u$ at any of the sampled points by means of a DMC side walk. It can be proved [20] that the naive way of squaring the weight leads to a biased estimate and that, to correctly estimate $u^{2}$, two independent paths have to be generated. A schematic representation of the method for the two quantities $\mathcal{V}$ and $\mathcal{U}$ is given in Fig. 2. The core of this algorithm is the imaginary time projection via short-time approximation employed in normal DMC simulations. Without introducing any ambiguity, we will therefore refer to the method using the acronym DMC. Examples of this kind of algorithm can be found in Refs. [21-23].

As far as the computation of $\mathcal{U}$ in Eq. (9) is concerned, we notice that the initial position of the walker, corresponding to an electron and the positron sitting on top of each other, represents a particularly pathological point for the short-time approximation of the Green's function normally employed in DMC [24]. There, the use of this approximation supplemented with the detailed balance condition is accurate only if the chosen time step is very small. Indeed we found, employing various time steps $\left(0.001-0.02\right.$ hartree $\left.^{-1}\right)$, that using the large ones has a dramatic effect on the value of $\mathcal{U}$ (see Fig. 3 ). In the limit of exact wave function $\mathcal{U}$ is identically 1 and this suggests that a good choice of the wave function can, as usual, largely improve the situation. When a good function is not available, it is possible to avoid the use of an extremely 


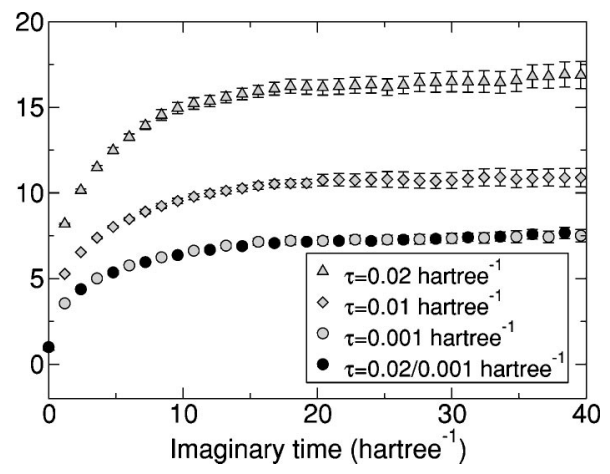

FIG. 3. Behavior of $\mathcal{U V}^{-1}$ as a function of the projection time. The system is $e^{+} \mathrm{He}$ with $\mathcal{R}=10 \mathrm{bohr}$ (see text for the meaning of $\mathcal{R}$ ). Black circles were obtained setting $\tau$ to 0.001 hartree $^{-1}$ for the first 100 steps and then to 0.02 hartree $^{-1}$.

small time step throughout the whole simulation by simply using a short-time step for the first few hundreds of steps, and increasing it after that the pathological coalescence position has been abandoned. An example of the benefit associated with this trick is shown in Fig. 3 where a particularly inaccurate trial function was used in order to enhance the effect.

\section{Scattering states}

The basic ideas for the application of QMC methods to scattering problems can be found elsewhere in the literature [25-28]. For the purpose of this paper it is enough to mention that the system can be viewed as enclosed in a spherical box of radius $\mathcal{R}$ which, in turns, specifies the form of the $s$-wave component of the wave function according to

$$
\Psi(x)=\Psi_{\mathrm{el}}\left(x_{\mathrm{el}}\right) \frac{\sin \left[k\left(r_{p}-\mathcal{R}\right)\right]}{r_{p}} .
$$

The diffusion Monte Carlo method is particularly tailored to deal with low-energy processes for which the $s$-wave component gives the only relevant contribution. Our discussion therefore is centered on this particular partial wave.

The only issue in extending the algorithm introduced in the preceding section to scattering states is represented by the unusual (for QMC simulations) boundary condition imposed on the wave function. The standard QMC normalization corresponds to considering a state normalized to one inside the simulation box and it is clearly useless when the expectation value requires a different constraint like that of Eq. (4).

To illustrate how this difficulty can be easily overcome let us call the correctly normalized function $Y$ and the function sampled during the simulation $\Psi$. We need to find the factor $\mathcal{N}$ such that $Y=\mathcal{N}^{1 / 2} \Psi . \mathcal{N}$ can be more usefully expressed as

$$
\mathcal{N}=\frac{\int_{\mathcal{Q}}|\Upsilon|^{2} d \mu}{\int_{\mathcal{Q}}|\Psi|^{2} d \mu},
$$

where $\mathcal{Q}$ can be any domain in configurational space. This leads to a possible correct estimator for $w$ as

$$
w[\Psi]=\frac{\int|\Phi|^{2} d \nu}{\int_{\mathcal{Q}}|\Psi|^{2} d \mu} \mathcal{F}(\mathcal{Q}, k),
$$

where $\mathcal{F}(\mathcal{Q}, k)=\int_{\mathcal{Q}}|Y|^{2} d \mu$. Equation (8) is thus straightforwardly modified to the close analog

$$
w\left[\Psi_{0}\right]=\frac{\int d \nu\left|\Phi_{0}\right|^{2}}{\int d \nu\left|\Phi_{T}\right|^{2}} \frac{\int d \nu\left|\Phi_{T}\right|^{2}}{\int_{\mathcal{Q}} d \mu\left|\Psi_{T}\right|^{2}} \frac{\int_{\mathcal{Q}} d \mu\left|\Psi_{T}\right|^{2}}{\int_{\mathcal{Q}} d \mu\left|\Psi_{0}\right|^{2}} \mathcal{F}(\mathcal{Q}, k) .
$$

The second factor $\int d \nu\left|\Phi_{T}\right|^{2}\left(\int_{\mathcal{Q}} d \mu\left|\Psi_{T}\right|^{2}\right)^{-1}$ is computed during a normal $\mathrm{VMC}$ simulation estimating the denominator as the number of configurations fallen in the domain $\mathcal{Q}$. The third factor $\int_{\mathcal{Q}} d \mu\left|\Psi_{T}\right|^{2}\left(\int_{\mathcal{Q}} d \mu\left|\Psi_{0}\right|^{2}\right)^{-1}$ is estimated constraining the paths of the variational random walk in the domain $\mathcal{Q}$ (a move is rejected when a walker exits $\mathcal{Q}$ ). Of course, the DMC side paths must not obey this constraint.

It is only left to find a suitable domain where $\mathcal{F}(\mathcal{Q}, k)$ can be computed analytically. Choosing this domain such that the $s$-wave asymptotic form [obtained partial-wave expanding Eq. (4)]

$$
\Upsilon(x)=\Upsilon_{\mathrm{el}}\left(x_{\mathrm{el}}\right) \frac{\sin \left[k r_{p}+\delta(k)\right]}{k r_{p}}
$$

holds at every point, makes this task extremely simple. If $\mathcal{Q}$ is chosen as the direct product of the entire threedimensional space for each electron and a spherical crown, whose external and internal radii are, respectively, $\mathcal{R}$ and $\mathcal{R}-\Delta R$, for the positron, one gets

$$
\mathcal{F}(\mathcal{Q}, k)=\frac{2 \pi}{k^{3}}[k \Delta R-\sin (k \Delta R) \cos (k \Delta R)],
$$

which completes the computation of every term in Eq. (17).

\section{TESTS}

The wave functions used in the calculations were mainly products of exponentials of Pade's approximants $\mathcal{J}(r)$,

$$
\mathcal{J}(r)=\exp \left(\frac{a r+b r^{2}}{1+c r}\right)
$$

where $a$ was fixed in order to exactly satisfy the particleparticle cusp condition and $b$ was sometimes set to 0 . When this latter constraint is applied, the function will be called $\mathcal{J}^{\prime}$. The following results are obtained without extensively optimizing the variational parameters of the wave functions. Our aim in doing this is to estimate the performance of the proposed DMC estimator in general cases where accurate wave functions might not be available. 
TABLE I. $\left\langle\delta_{e p}\right\rangle$ values.

\begin{tabular}{lcc}
\hline \hline & \multicolumn{1}{c}{ VMC/DMC $^{\mathrm{a}}$} & Other \\
\hline $\mathrm{Ps}^{-}$ & $0.015078(2) / 0.02081(5)$ & $0.020733198^{\mathrm{b}}$ \\
$\mathrm{PsH}$ & $0.023678(3) / 0.02476(6)$ & $0.0244611^{\mathrm{c}}$ \\
\hline \hline
\end{tabular}

${ }^{a} \mathrm{DMC}$ refers to the technique exposed in the text where DMC paths originate from a backbone VMC path.

${ }^{\mathrm{b}}$ References [29-31], exact value from three independent calculations.

${ }^{\mathrm{c}}$ Reference [33], SVM calculation with 1600 basis functions.

\section{A. Bound states}

As test cases, we chose to compute the annihilation properties for the ground state of the $\mathrm{Ps}^{-}$and the $\mathrm{PsH}$ systems. The properties of these two systems have been computed using explicitly correlated wave functions by several groups [29-31] and, nowadays, are known with sufficient accuracy to be used as a reference. Table I summarizes the values obtained for these two systems together with the reference values.

In all considered cases the variational estimate was computed using the simple and exactly normalizable $\xi^{\prime}\left(r_{1 p}\right)$ $=\exp \left(-0.5 r_{1 p}\right)$. Using a similar form for $\xi\left(r_{p}\right)$ leads to a variance one order of magnitude larger.

As $\mathrm{Ps}^{-}$is concerned, the trial wave function is

$$
\Psi_{\mathrm{Ps}^{-}}=\left(1+P_{12}\right)\left[\mathcal{J}_{12}^{\prime}\left(r_{12}\right) \mathcal{J}_{1 p}\left(r_{1 p}\right) \mathcal{J}_{2 p}\left(r_{2 p}\right)\right]
$$

where $P_{12}$ is the exchange operator between electron 1 and 2. Our wave function has a variational energy of $-0.252360(5)$ hartree to be compared with the best variational estimate of -0.262005 hartree [29]. The behavior of $\mathcal{U} \mathcal{V}^{-1}$ is reported in Fig. 4 and the VMC and DMC $\left\langle\delta_{e p}\right\rangle$ values are reported in Table I. Reference values of $\left\langle\delta_{e p}\right\rangle$ [29-31] agree up to eight digits and for the accuracy of our calculation can be considered as exact. Our final value of $\left\langle\delta_{e p}\right\rangle$ is $0.37 \%$ higher than the exact one, the difference being within a $2 \sigma$ interval centered on the mean value. This estimate can be considered statistically exact even though at this level of accuracy the time step bias could play a role in determining small discrepancies. A time step bias analysis was not pursued in this work.

For the case of $\mathrm{PsH}$ the wave function was chosen as

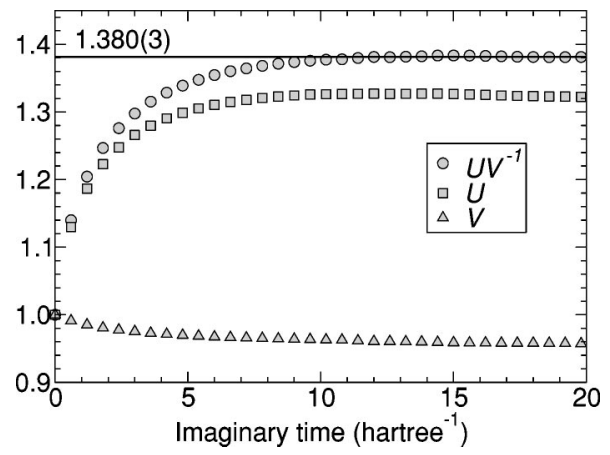

FIG. 4. $\mathcal{U}, \mathcal{V}$, and $\mathcal{U} \mathcal{V}^{-1}$ for $\mathrm{Ps}^{-}$as a function of the projection time. Error bars are smaller than the symbol size.

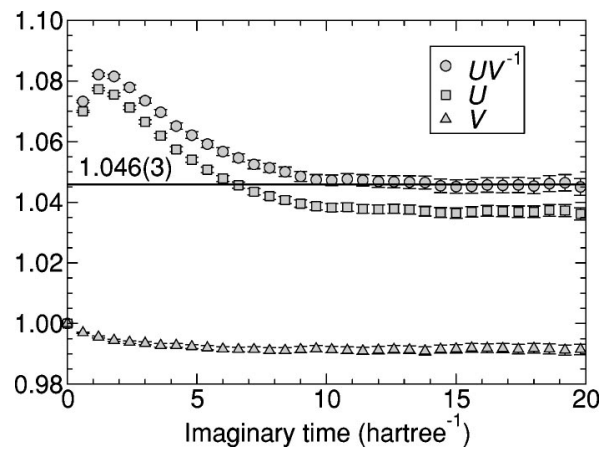

FIG. 5. $\mathcal{U}, \mathcal{V}$, and $\mathcal{U} \mathcal{V}^{-1}$ for PsH as a function of the projection time.

$$
\begin{aligned}
\Psi_{\mathrm{PsH}}= & \left(1+P_{12}\right)\left[\mathcal{J}_{1}\left(r_{1}\right) \mathcal{J}_{2}\left(r_{2}\right) \mathcal{J}_{12}^{\prime}\left(r_{12}\right)\right. \\
& \left.\times \mathcal{J}_{1 p}\left(r_{1 p}\right) \mathcal{J}_{2 p}^{\prime}\left(r_{2 p}\right) \mathcal{J}_{p}\left(r_{p}\right)\right]
\end{aligned}
$$

This wave function has a variational energy of -0.784620 (3) hartree, to be compared with the best variational estimate of -0.7891967 hartree [32]. This energy value is also very close to the value that one gets using a linear expansion of five correlated functions [16], but with a more immediate physical interpretation and less parameters. The behavior of $\mathcal{U}, \mathcal{V}$, and $\mathcal{U} \mathcal{V}^{-1}$ as a function of the projection time is reported in Fig. 5. The $\left\langle\delta_{p e}\right\rangle$ value computed using DMC is $1.2 \%$ higher than that of Ref. [33]. This last estimate, $\delta_{e p}=0.0244611$, seems converged up to the fifth decimal place and suggests that our value is likely to be affected by time step bias. As for $\mathrm{Ps}^{-}$no time step bias analysis was performed in this work.

\section{B. Scattering states}

The variational estimate for the scattering calculations was carried out employing $\xi(r)=r^{-1} \sin [(\pi / \mathcal{R})(r-\mathcal{R})]$ for both hydrogen and helium. It must be pointed out that the projection time required to eliminate all the excited-state components for these systems scales proportionally to $\mathcal{R}^{2}$. Therefore, it might become computationally expensive to run an entire simulation with a small time step when $\mathcal{R}$ is large. For this reason we employed the fairly large $\tau=0.02$ hartree $^{-1}$ to compute momenta and $\mathcal{V}$ 's. The effect that this large $\tau$ has on the value of $\mathcal{U}$ (see Sec. III B) was mitigated performing the first 100 steps of the simulations using $\tau$ $=0.001$. To see if this procedure was affected by time step bias, the simulation was repeated this time with a constant $\tau=0.001$ hartree $^{-1}$ for one of the small boxes $(\mathcal{R}$ $=15 \mathrm{bohr}$ ). The results of the two simulations were found statistically equal. At this point it is also worth stressing that an accurate value for the momentum can be obtained only if the energy of the target is computed with the same time step used during the simulation of the scattering state. In all cases the normalization coefficient $\mathcal{N}$ was computed using $\Delta R$ $=\mathcal{R} / 2$ (see the end of Sec. III C for the definition of $\Delta R$ ). The results were always found statistically equivalent to those obtained employing a smaller $\Delta R$.

The trial wave function for $e^{+} \mathrm{H}$ was 


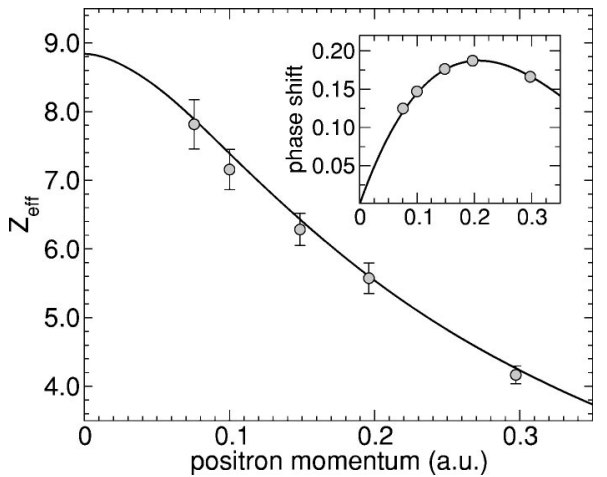

FIG. 6. Scattering of a positron off $\mathrm{H}$ ( $s$-wave component). $Z_{\mathrm{eff}}$ as a function of the positron momentum. Circles represent our estimates. The continuous line is taken from Refs. [34,35]. The inset reports the corresponding phase shifts and the continuous line is taken from Ref. [36].

$$
\Psi_{e^{+} \mathrm{H}}=\mathcal{J}_{1}\left(r_{1}\right) \mathcal{J}_{1 p}^{\prime}\left(r_{1 p}\right) \mathcal{J}_{p}^{\prime}\left(r_{p}\right) \frac{\sin \left[k\left(r_{p}-\mathcal{R}\right)\right]}{r_{p}},
$$

where $k$ was fixed to the free-particle value $k=\pi / \mathcal{R}$. In Fig. 6 the dependence of $Z_{\text {eff }}$ as a function of the momentum is reported. Values are also reported in Table II together with the radius of the boxes employed. In the $Z_{\text {eff }}$ graph the line represents the values given in Ref. [34] combined with the low-momentum values of Ref. [35]. Reference [34] reports an extensive study of this system using close coupling expansions for angular momentum up to $J=4$. Reference [35] presents a semiempirical method that provides a twoparameter description of scattering and annihilation. We used the values of this model to represent the low-energy part of $Z_{\text {eff }}(k<0.06$ a.u.). Points at $k>0.09$ a.u. are those of Ref. [34]. In this range of energy these data are consistent with our QMC values. Figure 6 contains also an inset with phase shifts computed in the same range of energy, while the continuous line is taken from Ref. [36].

Finally we consider the case of $e^{+} \mathrm{He}$. The trial function employed had the form

$$
\begin{aligned}
\Psi_{e^{+} \mathrm{He}}= & \left(1+P_{12}\right)\left[\mathcal{J}_{1}\left(r_{1}\right) \mathcal{J}_{2}\left(r_{2}\right) \mathcal{J}_{12}^{\prime}\left(r_{12}\right) \mathcal{J}_{1 p}^{\prime}\left(r_{1 p}\right)\right. \\
& \left.\times \mathcal{J}_{2 p}^{\prime}\left(r_{2 p}\right) \mathcal{J}_{p}^{\prime}\left(r_{p}\right)\right] \frac{\sin \left[k\left(r_{p}-\mathcal{R}\right)\right]}{r_{p}} .
\end{aligned}
$$

The results are reported in Table III and plotted in Fig. 7 where they are compared to those of Ref. [37]. The latter values were computed using a wave function that includes

TABLE II. $Z_{\text {eff }}$ and phase shift $\delta$ for $e^{+} \mathrm{H}$.

\begin{tabular}{lccc}
\hline \hline $\mathcal{R}$ (bohr) & $k$ (a.u.) & $Z_{\text {eff }}$ & $\delta$ \\
\hline 10 & $0.2975(2)$ & $4.2(1)$ & $0.166(2)$ \\
15 & $0.1969(2)$ & $5.6(2)$ & $0.188(3)$ \\
20 & $0.1483(2)$ & $6.3(2)$ & $0.176(4)$ \\
30 & $0.0998(1)$ & $7.2(3)$ & $0.148(3)$ \\
40 & $0.0754(1)$ & $7.8(4)$ & $0.126(4)$ \\
\hline \hline
\end{tabular}

TABLE III. $Z_{\text {eff }}$ and phase shift $\delta$ for $e^{+} \mathrm{He}$.

\begin{tabular}{lccc}
\hline \hline $\mathcal{R}$ (bohr) & $k$ (a.u.) & $Z_{\text {eff }}$ & $\delta$ \\
\hline 10 & $0.31152(5)$ & $3.16(2)$ & $0.0264(4)$ \\
15 & $0.20676(6)$ & $3.50(3)$ & $0.0402(8)$ \\
20 & $0.15517(8)$ & $3.70(5)$ & $0.038(1)$ \\
30 & $0.1036(1)$ & $3.87(5)$ & $0.032(3)$ \\
40 & $0.0777(1)$ & $3.91(7)$ & $0.030(5)$ \\
\hline \hline
\end{tabular}

two distinct contributions. The first describes the asymptotic form of the scattering wave function (when the positron is far from the helium atom). The second is a 502-terms expansion (identical to that employed in Ref. [39]) that aims to describe the short-range correlation between the positron and the particles constituting the helium atom. Our estimates and those of Ref. [37] are in good agreement even though at small momenta ours suffer for a quite large statistical noise. The inset in Fig. 7 reports the phase shifts in the same range of energies, while the continuous line was taken from Ref. [36]. This highlights, such as for the scattering of the positron off $\mathrm{H}$, the good agreement of our phase shifts with very accurate literature data.

\section{CONCLUSIONS}

In this work, an exact scheme to evaluate the positronelectron annihilation rate in bound and scattering states has been introduced. It has been tested using four small systems for which accurate results computed by other methods are available. In every case the agreement between those values and our estimates is quite good.

When compared with algebraic approaches employing basis set expansions, this Monte Carlo algorithm (as every Monte Carlo algorithm) has the advantage of being immediately extensible to deal with systems containing more particles than those used in these present test calculations. For instance, DMC calculations on 12 electrons and a positron have already been performed [12] and, as far as we know, no other method is capable of treating explicitly this number of

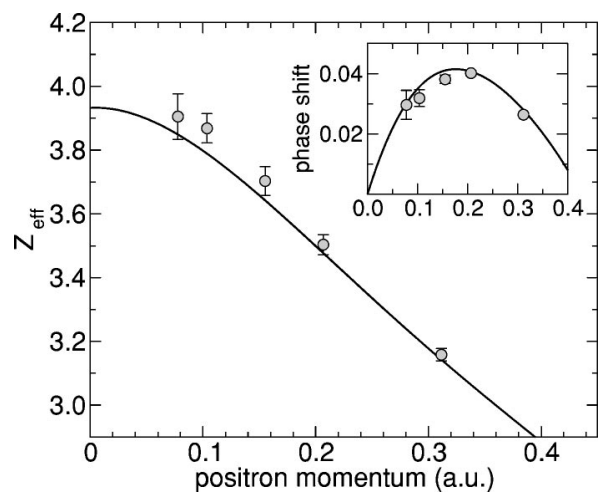

FIG. 7. Scattering of a positron off He ( $s$-wave component). $Z_{\text {eff }}$ as a function of the positron momentum. Circles represent our estimates. The continuous line is taken from Ref. [37]. The inset reports the corresponding phase shifts and the continuous line is taken from Ref. [38]. 
particles with comparable accuracy. This fact paves the way to accurately estimate the annihilation rate of systems such as $\mathrm{PsF}$ and $\mathrm{PsCl}$. We expect the present algorithm to perform well without changes for the aforementioned systems.

Compared to other estimators already proposed in the context of VMC, the one introduced here is free from the "divergent variance" feature that plagues some of them. The central quantity, the ratio $\Phi^{(i)} / \Psi$, can be seen as the ratio of the wave function after and before the move $r_{p} \rightarrow r_{i}$. Routines for an efficient computation of this ratio are already present in QMC codes moving one electron at a time. The computation of the whole sum in Eq. (3) requires therefore an effort that scales linearly with the number of particles because of the requirement of moving the positron on top of each electron. Often one can exploit the permutational symmetry of the problem and indeed reduce the sum to the computation of only one or few more of the addends in Eq. (3). For the case of a wave function built starting from a restricted Hartree-Fock determinant, a correct estimate can be always computed using only one electron provided that the sampling is allowed also in regions where the wave function is negative. Of course time can also be saved if this quantity is not computed at each step. It remains to understand if the simple choices made in this work for the functions $\xi$ and $\xi^{\prime}$ will remain a valid possibility for larger systems. More generally it is left to prove that, for many particle systems, it is indeed possible to find $\xi$ or $\xi^{\prime}$ such that the variance of the estimator will continue to remain as small as that observed in this work.

As far as the exact algorithm is concerned, the main improvement with respect to other approaches is the possibility of going beyond the mixed estimate typical of DMC simulations. This, of course, at the price of a more computationally demanding algorithm. Unfortunately the nonlocal estimator for the $\delta$ function [16] involves differential operators and therefore cannot be combined with the forward walking or the reptation algorithm [40]. Techniques based on extrapolation could instead benefit from the possibility of sampling the square of the exact wave function as done in the reptation algorithm. This possibility remains to be explored.

Finally we want to comment the general performance of the DMC algorithm for scattering states. As already stated DMC is tailored to work with low-energy processes. However, as clearly shown by Fig. 7, the performance of the method degrades at very low momenta because of the necessity of employing large radii $\mathcal{R}$. This pathological behavior can be cured through the usage of more sophisticated sampling techniques. Of particular benefit should be the possibility of imposing different boundary condition on $\mathcal{R}$ [i.e., fixing the logarithmic derivative to any desired value instead of imposing $\Psi(\mathcal{R})=0$ ] and of employing correlated sampling to determine more precisely the value of the momentum. Both these topics will be the subject of future investigations.

\section{ACKNOWLEDGMENTS}

S.C. would like to thank David Ceperley for pointing out the possibility of changing the time step during the DMC projection runs. M.M. would like to thank Jim Mitroy for many useful discussions on positron scattering off atomic targets.
[1] O.E. Mogensen, Positron Annihilation in Chemistry (SpringerVerlag, Berlin, 1995).

[2] S.J. Gilbert, L.D. Barnes, J.P. Sullivan, and C.M. Surko, Phys. Rev. Lett. 88, 043201 (2002).

[3] G.F. Gribakin and J. Ludlow, Phys. Rev. Lett. 88, 163202 (2002).

[4] P. Van Reeth and J.W. Humberston, J. Phys. B 31, L231 (1998).

[5] A. Igarashi, M. Kimura, and I. Shimamura, Phys. Rev. Lett. 89, 123201 (2002).

[6] I.A. Ivanov and J. Mitroy, J. Phys. B 33, L831 (2000).

[7] D. Bressanini, M. Mella, and G. Morosi, J. Chem. Phys. 108, 4756 (1998).

[8] D. Bressanini, M. Mella, and G. Morosi, J. Chem. Phys. 109, 1716 (1998).

[9] D. Bressanini, M. Mella, and G. Morosi, J. Chem. Phys. 109, 5931 (1998).

[10] M. Mella, G. Morosi, and D. Bressanini, J. Chem. Phys. 111, 108 (1999).

[11] M. Mella, G. Morosi, D. Bressanini, and S. Elli, J. Chem. Phys. 113, 6154 (2000).

[12] M. Mella, M. Casalegno, and G. Morosi, J. Chem. Phys. 117, 1450 (2002).

[13] A.M. Frolov, S.I. Kryuchkov, and V.H. Smith, Jr., Phys. Rev. A 51, 4514 (1995).
[14] M. Mella, S. Chiesa, and G. Morosi, J. Chem. Phys. 116, 2852 (2002).

[15] S.A. Alexander and R.L. Coldwell, J. Chem. Phys. 103, 2572 (1995).

[16] D. Bressanini, M. Mella, and G. Morosi, Phys. Rev. A 57, 1678 (1998).

[17] P.A. Fraser, Adv. At. Mol. Phys. 4, 63 (1968).

[18] The region considered in this case is the set of configurations where the positron is on top of one of the electrons. This region has null measure.

[19] M. Caffarel and P. Claverie, J. Chem. Phys. 88, 1088 (1988).

[20] P.J. Reynolds, J. Chem. Phys. 92, 2118 (1990).

[21] R.N. Barnett, P.J. Reynolds, and W.A. Lester, Jr., J. Comput. Phys. 96, 258 (1991).

[22] R.N. Barnett, P.J. Reynolds, and W.A. Lester, Jr., J. Chem. Phys. 96, 2141 (1992).

[23] D.M. Ceperley and B.J. Alder, Phys. Rev. A 31, 1999 (1985).

[24] C.J. Umrigar, M.P. Nightingale, and K.J. Runge, J. Chem. Phys. 99, 2865 (1993).

[25] Y. Alhassid and S.E. Koonin, Ann.Phys. 155, 108 (1984).

[26] J. Carlson, V.R. Pandharipande, and R.B. Wiringa, Nucl. Phys. A 424, 47 (1984).

[27] J. Shumway and D.M. Ceperley, Phys. Rev. B 63, 165209 (2001). 
[28] S. Chiesa, M. Mella, and G. Morosi, Phys. Rev. A 66, 042502 (2002).

[29] A.M. Frolov, Phys. Rev. A 60, 2834 (1999).

[30] R. Krivec, V.B. Mandelzweig, and K. Varga, Phys. Rev. A 61, 062503 (2000).

[31] G.W.F. Drake, M.M. Cassar, and R.A. Nistor, Phys. Rev. A 65, 054501 (2002).

[32] Z.-C. Yan and Y.K. Ho, Phys. Rev. A 59, 2697 (1999).

[33] J. Usukura, K. Varga, and Y. Suzuki, Phys. Rev. A 58, 1918 (1998).

[34] G.G. Ryzhikh and J. Mitroy, J. Phys. B 33, 2229 (2000).
[35] J. Mitroy and I.A. Ivanov, Phys. Rev. A 65, 042705 (2002).

[36] J. Mitroy, L. Berge, and A. Stelbovics, Phys. Rev. Lett. 73, 2966 (1994).

[37] P. Van Reeth, J.W. Humberston, K. Iwata, R.G. Greaves, and C.M. Surko, J. Phys. B 29, L465 (1996).

[38] P. Van Reeth and J.W. Humberston, J. Phys. B 32, 3651 (1999).

[39] A.M. Frolov and V.H. Smith, Jr., Phys. Rev. A 56, 2417 (1997).

[40] S. Baroni and S. Moroni, Phys. Rev. Lett. 82, 4745 (1999). 